Mirai. Estudios Japoneses

ISSN-e: 2531-145X

http://dx.doi.org/10.5209/mira.67233

\title{
Los principios para el desarrollo de la Inteligencia Artificial en Japón y las avenidas para la cooperación con la Unión Europea ${ }^{1}$
}

\author{
Ana Gascon Marcen²
}

Resumen. En este trabajo, se analizarán los principios que ha adoptado Japón recientemente para asegurarse de que el desarrollo de la Inteligencia Artificial se lleva a cabo de una manera que beneficie a las personas y a la sociedad. Estas directrices tienen como objetivo fomentar el diálogo a nivel internacional por lo que se estudiará también la visión europea como el socio natural de Japón en la materia. Finalmente, se propondrán opciones para la cooperación entre los dos, para terminar con unas breves conclusiones.

Palabras clave: Japón; Unión Europea; Inteligencia Artificial; principios éticos

\section{[en] The principles for the development of Artificial Intelligence in Japan and the avenues for cooperation with the European Union}

\begin{abstract}
In this paper, the principles that Japan has recently adopted to ensure that the development of Artificial Intelligence is carried out in a way that benefits people and society are assessed. These guidelines aim to promote dialogue at the international level, so the European vision as Japan's natural partner in the matter is also studied. Finally, options for cooperation between the two are proposed, to end with some brief conclusions.
\end{abstract}

Keywords: Japan; European Union; Artificial Intelligence; ethical principles

Sumario. 1. Introducción; 2. Japón y la gobernanza de la Inteligencia Artificial: 2.1 La situación en Japón; 2.2 Japón y los principios que deben gobernar la Inteligencia Artificial; 3. La Unión Europea y la gobernanza de la Inteligencia Artificial: 3.1 La situación en la Unión Europea; 3.2 La Unión Europea y los principios que deben gobernar la Inteligencia Artificial; 4. Posibilidades de cooperación entre Japón y la Unión Europea; 5. Conclusiones; 6. Bibliografía.

Cómo citar: Gascon Marcen, A. Los principios para el desarrollo de la Inteligencia Artificial en Japón y las avenidas para la cooperación con la Unión Europea, en Mirai. Estudios Japoneses, 4, 2020, 23-34.

\section{Introducción}

El término "inteligencia artificial" fue acuñado por el científico estadounidense, John McCarthy, en 1955, quien consideraba que cada aspecto del aprendizaje o cualquier otra característica de la inteligencia pueden describirse en principio con tanta precisión que se podría hacer una máquina para simularlos. Así, se crearían máquinas que usen el lenguaje, formen abstracciones y conceptos, resuelvan tipos de problemas hasta entonces reservados para los humanos y se automejoren ${ }^{3}$. En los últimos años, la capacidad de computación de los ordenadores, los nuevos algoritmos y la recogida de inmensas cantidades de datos que pueden ser procesadas por ellos (big data) han hecho que estas predicciones se confirmen en gran medida.

Según la Comisión Europea, el término "inteligencia artificial" se aplica a los sistemas que manifiestan un comportamiento inteligente, pues son capaces de analizar su entorno y pasar a la acción -con cierto grado de autonomía- con el fin de alcanzar objetivos específicos ${ }^{4}$.

Estos sistemas están cada vez más presentes en nuestra vida diaria, muchos de ellos incorporados en nuestros propios teléfonos móviles como asistentes de voz, programas de análisis de imágenes, motores de búsqueda, sistemas

\footnotetext{
Este artículo se ha realizado con el apoyo del proyecto de investigación RTI2018-097460-B-I00: Entornos laborales robotizados. Estudio de las propuestas japonesas para la sostenibilidad del gasto público y su aplicabilidad en la UE.

Universidad de Zaragoza; angascon@unizar.es

McCarthy, J. et al. (1955).

Comunicación de la Comisión al Parlamento Europeo, al Consejo Europeo, al Consejo, al Comité Económico y Social Europeo y al Comité de las Regiones Inteligencia artificial para Europa, COM(2018) 237 final, p. 1. Para un estudio más pormenorizado sobre la definición de la "inteligencia artificial", High-Level Expert Group on Artificial Intelligence (8 de abril de 2019).
} 
de reconocimiento facial y de voz. Pueden mejorar desde los diagnósticos médicos ${ }^{5}$, la gestión del transporte urbano ${ }^{6} \mathrm{o}$ la agricultura 7 . Pero también pueden poner en serio riesgo el disfrute de nuestros derechos humanos ${ }^{8}$ desde la libertad de expresión o asociación, la privacidad, el acceso a un recurso efectivo ${ }^{9}$, la prohibición de la discriminación ${ }^{10}$, hasta nuestros derechos sociales ${ }^{11}$.

Japón es un país altamente digitalizado, que considera prioritario el desarrollo de la inteligencia artificial, pero que, al ser una democracia que respeta el estado de derecho y los derechos humanos, no puede permitir que esta innovación se lleve a cabo sin ningún tipo de control. Además, es consciente de que ésta es una discusión que debe realizarse a nivel internacional y ha decidido ser uno de los actores que impulse tales debates.

En este trabajo, se analizará la labor de Japón en lo relativo a la gobernanza de la Inteligencia Artificial (apartado 2), ya que es uno de los Estados más avanzados en esa materia en el mundo, incorporando también la visión europea (apartado 3), al ser uno de los socios naturales de Japón en este tema, y, finalmente, se estudiarán las avenidas para la cooperación entre los dos (apartado 4), para terminar con unas breves conclusiones.

\section{Japón y la gobernanza de la Inteligencia Artificial}

\subsection{La situación en Japón}

Japón se encuentra décimo en una escala que califica a los gobiernos de 194 países de acuerdo con su preparación para usar inteligencia artificial (IA) en la prestación de servicios públicos ${ }^{12}$. En el Artificial Intelligence Index de Stanford, aparece como el cuarto país en lo que se refiere a la publicación de artículos sobre IA y el segundo en publicación de patentes y como mercado de robots industriales, aunque a mucha distancia de los primeros en ambas categorías, que son respectivamente Estados Unidos y China ${ }^{13}$.

Uno de los problemas de Japón es el de la financiación de la innovación, porque ésta se había reducido mucho en los años noventa quedándose estancada ${ }^{14}$, algo que parece contraproducente en una sociedad como la actual. Esto supuso un descenso en la relevancia de su investigación a nivel internacional frente al ascenso de sus vecinos China y Corea del Sur, que llevó a una reciente modificación del sistema de asignación de la financiación pública que no había sufrido una reforma de calado en los últimos cincuenta años ${ }^{15}$. Esto ha ido acompañado de subidas de la financiación pública desde 2018, en concreto un $10 \%$ en $2019^{16}$, y el lanzamiento del Programa Moonshot que busca estimular la innovación disruptiva en Japón, implementando desafíos de I+D basados en ideas que no son solo extensiones de la tecnología convencional ${ }^{17}$.

Otro de los problemas de Japón es la falta de científicos de datos, por lo que ha decidido crear cursos introductorios de IA en la universidad que son obligatorios ${ }^{18}$. Esto responde a una sensación de urgencia compartida por muchos expertos que consideran que Japón estaba a la vanguardia de la tecnología global, pero en unos pocos años ha perdido su posición ${ }^{19}$.

No obstante, Japón sigue contando con algunas ventajas como sus infraestructuras, por ejemplo, se aceleró la implantación de la tecnología 5G de cara a las olimpiadas de 2020. En 2025, se prevé que del total de conexiones móviles en el mundo un 15\% serán con 5G, llevando la delantera Corea del Sur (59\%), Estados Unidos (50\%) y Japón (48\%), mientras China y Europa (ambos con un 29\%) se quedarán atrás ${ }^{20}$. La tecnología $5 \mathrm{G}$ facilitará la capacidad y rapidez de las conexiones promoviendo que más aparatos se conecten e intercambien datos, de manera más rápida y con menos latencia, fomentando así el desarrollo del Internet de las Cosas (IoT por sus siglas en inglés).

En 2016, Japón publicó su V Plan Básico de Ciencia y Tecnología (2016-2021) que establece el objetivo para Japón de liderar la transición de la "industria 4.0"21 a la "sociedad 5.0"22 y construir una sociedad superinteligente. Japón consideraba

Park, S. H./Han. K. (2018) y Dilsizian, S. E./Siegel, E. L. (2014).

Jin, X./Wang, D. (2008).

Patrício, D. I./Rieder, R. (2018).

Raso, F. et al. (2018).

www.rm.coe.int (2017).

Borgesius, F. Z. (2018).

Alston, P. (2019)

2 Miller H. / Stirling, R. (2019): 32. La puntuación general se extrae de cuatro categorías: gobierno; infraestructura y datos; habilidades y educación; y servicios públicos y gubernamentales. Los datos se derivan de una variedad de recursos, desde su propia investigación sobre estrategias de IA, hasta bases de datos como el número de nuevas empresas registradas de IA en Crunchbase, hasta el Índice de Desarrollo de Administración Electrónica de las Naciones Unidas.

13 Perrault, R. et al. (2019).

14 www.oecd.org (2018).

15 Hornyak T. (2017). Las empresas también están aumentando su inversión en investigación, aunque probablemente no con la rapidez necesaria. Oikawa, A. (2019).

16 Jacob, M. (2019).

17 www8.cao.go.jp (2018).

18 Yamashita, M. (2019).

Inagaki, K. (2019).

www.gsmaintelligence.com (2019): 14.

21 Sería la cuarta revolución industrial, las anteriores serían la primera que se dio con la mecanización, energía hidráulica y energía eléctrica; la segunda con la producción en masa, la línea de montaje, y la electricidad; la tercera con la informática y la automatización.

22 Ortega Klein, A. (2019). Sigue a la sociedad de caza, la sociedad agrícola, la sociedad industrial y la sociedad de la información. 
que en todo el mundo había iniciativas que utilizan redes e Internet de las Cosas para mejorar la fabricación, pero ellos querían ir más allá y que su uso se extienda a otros campos para promover el crecimiento económico, pero también la formación de una sociedad sana y la transformación social. De esa manera, los frutos de la ciencia y la tecnología penetrarían en todo tipo de campos y esferas. Promueven una visión del futuro que se caracteriza por la integración sofisticada del ciberespacio con el espacio físico y una mayor coordinación global con una visión más holística ${ }^{23}$.

En la Estrategia Integrada de Innovación multianual de Japón lanzada en 2017, dentro de los campos en los que es imperativo mejorar para el país se cita como el primero la tecnología basada en IA. En esta área se fijaban tres objetivos: establecer una base de recursos humanos, promover un desarrollo tecnológico estratégico y formular unos principios de desarrollo de la inteligencia artificial centrados en los humanos para debatirlos a nivel internacional (lo que se desarrollará en el apartado siguiente) $)^{24}$.

En 2016, Japón estableció un Consejo de Estrategia de Tecnología de Inteligencia Artificial que publicó la Estrategia de Tecnología de Inteligencia Artificial en $2017^{25}$, que tiene tres fases:

- Fase 1: utilización y aplicación de inteligencia artificial basada en datos desarrollada en varios dominios (hasta 2020);

- $\quad$ Fase 2: uso público de IA y datos desarrollados en varios dominios (hasta 2025-2030);

- $\quad$ Fase 3: ecosistema creado mediante la conexión de dominios multiplicadores ${ }^{26}$.

En 2018, el Gabinete adoptó la Declaración para ser la nación más avanzada del mundo en tecnologías de la información y el Plan básico para el avance de la utilización de datos del sector público y privado que también describe la política del Gobierno para avanzar en las tecnologías que utilizan IA y IoT.

En Japón no hay una norma general que regule la IA, pero sí que ha habido reformas legislativas para promover la innovación en este campo, como la reforma de la Ley de protección de la información personal para facilitar el uso de Big Data en $2015^{27}$. También son pioneros en la reforma del régimen de protección de los derechos de autor creando excepciones que faciliten la minería de textos y datos ${ }^{28}$ en 2009. Además, en Japón, se ha discutido mucho sobre los principios que deben regular el desarrollo de la IA, como se explica en el siguiente apartado.

\subsection{Japón y los principios que deben gobernar la Inteligencia Artificial}

Japón empezó a trabajar en esta materia en 2015 y la Conferencia hacia una Sociedad en Red basada en la IA ${ }^{29}$ publicó el proyecto de Directrices de Investigación y Desarrollo de IA ${ }^{30}$ en julio de 2017 para los desarrolladores e investigadores de IA que propugnaban los principios de colaboración, transparencia, controlabilidad, seguridad (tanto safety como security), privacidad, ética, asistencia al usuario y responsabilidad ${ }^{31}$. A éstas, se sumó en julio de 2018 , el proyecto de Principios de Utilización de la IA $^{32}$ para los usuarios de sistemas o servicios de IA y proveedores de datos con los principios de utilización adecuada, calidad de los datos, colaboración, seguridad, privacidad, dignidad humana e individual, autonomía, equidad, transparencia y responsabilidad. Estos proyectos presentan directrices y principios a tener en cuenta para reducir los riesgos asociados con los sistemas que usan IA, tales como la opacidad de los procesos y la pérdida de control, y poder aprovechar sus múltiples ventajas.

En diciembre de 2018, la Conferencia publicó los Principios Sociales de la IA centrada en el ser humano ${ }^{33}$. En ellos, se establece que es esencial respetar unos valores filosóficos básicos como son la dignidad, la diversidad y la inclusión, y sostenibilidad. Para asegurar que la IA se usa con fines positivos, es necesario preparar los diferentes niveles de la sociedad para responder tanto a nivel humano, de sistemas sociales, estructuras industriales, sistemas de innovación y gobernanza. En especial, se detalla que los principios sociales de desarrollo de la IA deben ser: centrados en las personas; la educación; la privacidad; la seguridad; la competencia leal; la equidad, responsabilidad y transparencia; y la innovación.

Los propios principios establecen que Japón debe compartir estas pautas con otros países y asumir el liderazgo del debate internacional y tratar de llegar a un consenso. Así, podría exportar el concepto de la sociedad 5.0 que apoya la realización de los Objetivos de Desarrollo Sostenible en el mundo y debe contribuir al desarrollo cooperativo y creativo de la comunidad internacional.

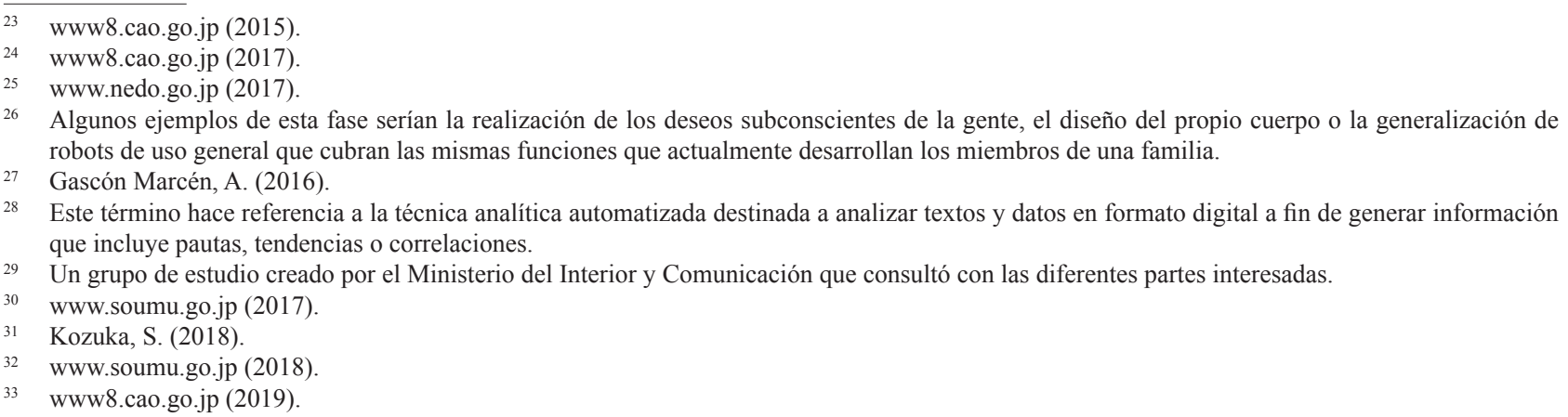


Japón ha aprovechado las ocasiones en las que ha sido anfitrión de foros internacionales para avanzar en este sentido. En la reunión de Ministros de Tecnologías de la Información y la Comunicación del G7 $7^{34}$ en 2016 celebrada en Takamatsu, propuso realizar un estudio sobre los impactos sociales y económicos de la IA en red a través de la colaboración, incluidas las organizaciones internacionales, y vincular esto con la discusión sobre los posibles principios en el desarrollo de la IA en red $^{35}$.

En la reunión del G20 $0^{36}$ de la que fue anfitrión en 2019, promovió la adopción de una declaración ministerial sobre comercio y economía digital ${ }^{37}$ que incluía un anexo con los Principios de IA del G20 ${ }^{38}$ que son: crecimiento inclusivo, desarrollo sostenible y bienestar; valores humanos e imparcialidad; transparencia y explicabilidad; solidez, seguridad y protección; y responsabilidad. Incluso consiguió que la Declaración final de los líderes asumiera la concepción japonesa de una IA centrada en las personas ${ }^{39}$. En concreto, los líderes se comprometieron con un enfoque centrado en el ser humano para la IA, y acogieron con beneplácito los Principios no vinculantes del G20 sobre IA, extraídos de la Recomendación del Consejo de la Organización para la Cooperación y el Desarrollo Económico sobre $\mathrm{IA}^{40}$. Es interesante conseguir una Declaración de esta naturaleza en el G20 porque forman parte de él países con una ideología diferente a la europea o japonesa como China o Arabia Saudita, por ejemplo. La cuestión es si esto tendrá alguna consecuencia práctica.

\section{La Unión Europea y la gobernanza de la Inteligencia Artificial}

\subsection{La situación en la Unión Europea}

Es difícil explicar la situación de la Unión Europea (UE) en lo que se refiere a la IA dada la disparidad que existe entre sus Estados miembros, cinco de ellos (Alemania, Finlandia, Suecia, Francia y Dinamarca) se encuentran entre los 10 primeros del Government Artificial Intelligence Readiness Index 2019, pero Croacia aparece en el puesto $62^{41}$. Actualmente, China publica tantos artículos sobre IA como todos los Estados europeos juntos, siendo Alemania el quinto país del mundo que más publica ${ }^{42}$.

En el año 2000, la UE se fijó como objetivo para 2010 convertirse en la economía basada en el conocimiento más competitiva y dinámica del mundo a través de la Estrategia de Lisboa. Es obvio que fracasó. Por eso, es importante no quedar también rezagados en lo que a la IA se refiere.

La IA ha figurado en los programas marco de investigación y desarrollo de la UE desde 2004. A través del programa de investigación e innovación Horizonte 2020 (2014-2017), se han invertido globalmente cerca de 1100 millones de euros en investigación e innovación relacionada con la IA. En 2018, la UE en su conjunto (sumando los sectores público y privado) se propuso incrementar esta inversión al menos hasta 20,000 millones de euros hasta finales de 2020, e invertir más de 20,000 millones de euros anuales durante la próxima década. La Comisión, por su parte, iba a aumentar las inversiones en IA a alrededor de 1,500 millones de euros hasta finales de 2020 (lo que representa un aumento del 70\%). Todo esto quedó fijado en su Estrategia para la Inteligencia Artificial de $2018^{43}$. Además, la UE facilitó la creación de una Alianza Europea de la Inteligencia Artificial para reunir a las empresas y las administraciones públicas.

Actualmente, la UE no tiene una norma que regule de manera general la $\mathrm{IA}^{44}$, pero sí algunas sectoriales. Un ejemplo es el Reglamento General de Protección de Datos ${ }^{45}$ cuyo artículo 22 establece que todo interesado tendrá

34 El Grupo de los Siete o G7 reúne periódicamente a los representantes de algunos de los países económicamente más importantes del mundo y que comparten una ideología similar: Alemania, Canadá, Estados Unidos, Francia, Italia, Japón y Reino Unido. La UE también está representada.

35 Millar, J. et al. (2018).

36 El Grupo de los 20 o G20 es un foro periódico en el que se reúnen los representantes de algunos de los Estados económicamente más relevantes del mundo: Alemania, Arabia Saudita, Argentina, Australia, Brasil, Canadá, China, Corea del Sur, Estados Unidos, Francia, India, Indonesia, Italia, Japón, México, Reino Unido, Rusia, Sudáfrica, Turquía y la UE.

37 Cuatro países relevantes (India, Egipto, Indonesia y Sudáfrica) no firmaron la Declaración, si bien como explica Greenleaf, G. (2019) esto no se debía a la parte dedicada a los principios de la IA, sino a la que se consagró al libre flujo de datos con confianza (Data Free Flow with Trust) creando la Osaka Track. Un marco para fomentar las negociaciones en el marco de la Organización del Comercio para facilitar el libre comercio electrónico acompañado de un libre flujo de datos con garantías tanto en lo relativo a la libre circulación de datos personales como a la ciberseguridad.

38 www.mofa.go.jp (2019).

39 www.consilium.europa.eu (2019b).

40 www.legalinstruments.oecd.org (2019).

No obstante, Clarke (2019) critica que esta Recomendación es muy limitada, porque según la metodología que ha desarrollado para medir el alcance de las diferentes declaraciones de principios que deben regir el desarrollo de la IA sólo obtiene un 20/50, mientras que la de la UE que se examinará en el apartado siguiente consigue un 37/50 al cubrir muchos más ámbitos.

41 Miller H./Stirling, R. (2019).

42 Perrault, R. et al. (2019): 5 y 24.

43 www.legalinstruments.oecd.org (2018).

44 Más allá de la UE, merece la pena mencionar, en el ámbito europeo, la creación de un Comité Ad Hoc sobre Inteligencia Artificial (CAHAI) por parte del Consejo de Europa para reflexionar sobre la posibilidad de negociar en su marco un acuerdo internacional sobre la materia desde la perspectiva de la protección de los derechos humanos.

Japón, como Estado observador de la organización, podría involucrase en la negociación, dado que ya participa de diversas formas en varios convenios del Consejo de Europa relacionados con las tecnologías de la información y la comunicación. Es parte del Convenio sobre ciberdelincuencia y observador del Comité Consultivo del Convenio para la protección de las personas con respecto al tratamiento automatizado de datos de carácter personal.

45 Reglamento (UE) 2016/679 del Parlamento Europeo y del Consejo, de 27 de abril de 2016, relativo a la protección de las personas físicas en lo que respecta al tratamiento de datos personales y a la libre circulación de estos datos y por el que se deroga la Directiva 95/46/CE (Reglamento general de protección de datos), DOUE L 119, 4.5.2016, pp. 1-88. 
derecho a no ser objeto de una decisión basada únicamente en el tratamiento automatizado, incluida la elaboración de perfiles, que produzca efectos jurídicos en él o le afecte significativamente de modo similar. A lo que se añade que el artículo 15 regula que el interesado tendrá derecho a obtener del responsable del tratamiento confirmación de si se están tratando o no datos personales que le conciernen y, en tal caso, derecho de acceso a la información sobre la existencia de decisiones automatizadas, incluida la elaboración de perfiles, e información significativa sobre la lógica aplicada, así como la importancia y las consecuencias previstas de dicho tratamiento para el interesado ${ }^{46}$.

La Presidenta de la Comisión Europea se comprometió en su programa a, en sus primeros 100 días en el cargo (empezó el 1 de diciembre de 2019), presentar propuestas de legislación para un enfoque europeo coordinado sobre las implicaciones éticas y humanas de la $\mathrm{IA}^{47}$.

\subsection{La Unión Europea y los principios que deben gobernar la Inteligencia Artificial}

En lo relativo a los desafíos jurídicos y legales que plantea el uso extendido de la IA, el Grupo Europeo de Ética de la Ciencia y de las Nuevas Tecnologías (grupo consultivo de la Comisión) publicó en marzo de 2018 una Declaración sobre inteligencia artificial, robótica y sistemas autónomos ${ }^{48}$.

Basándose en el trabajo de ese Grupo, la Comisión decidió reunir a todas las partes interesadas pertinentes en un Grupo de Expertos de Alto Nivel en Inteligencia Artificial, para contribuir al desarrollo de un proyecto de directrices éticas en este ámbito ${ }^{49}$. Estas directrices debían tratar cuestiones tales como el futuro del trabajo, la equidad, la seguridad, la protección, la inclusión social y la transparencia de los algoritmos. En términos más generales, en ellas se examina el impacto en los derechos fundamentales, en particular, la intimidad, la dignidad, la protección de los consumidores y la lucha contra la discriminación.

En 2018 se publicó un primer borrador para recibir comentarios y finalmente el 8 de abril de 2019 se presentaron las Directrices éticas para una IA fiable ${ }^{50}$. Su objetivo es conseguir una IA fiable que tiene tres componentes: debe ser lícita, es decir, cumplir todas las leyes y reglamentos aplicables; ha de ser ética, de modo que se garantice el respeto de los principios y valores éticos; y debe ser robusta ${ }^{51}$.

El Capítulo I trata de garantizar el propósito ético de la IA, estableciendo cuáles son los derechos, principios y valores fundamentales que debe cumplir. En ese sentido, la IA debe estar centrada en el ser humano y se debe desarrollar, implementar y utilizar con un propósito ético. Tiene que regirse por los derechos fundamentales, los valores sociales y los principios éticos de respeto de la autonomía humana, prevención del daño, equidad y explicabilidad. Es necesario prestar especial atención a las situaciones que involucran a grupos vulnerables o a situaciones con asimetrías de poder o información (como entre empleadores y empleados, o empresas y consumidores).

El Capítulo II desarrolla una guía concreta enumerando los requisitos para la IA fiable y ofreciendo una visión general de los métodos técnicos y no técnicos que se pueden utilizar para su implementación. Estos requisitos que deben incluirse desde la primera fase de diseño son: acción y supervisión humanas; solidez técnica y seguridad; gestión de la privacidad y de los datos; transparencia; diversidad, no discriminación y equidad; bienestar ambiental y social; y rendición de cuentas. Debe hacerse un esfuerzo por facilitar la capacidad de auditoría de los sistemas de inteligencia artificial, especialmente en contextos o situaciones críticas, además, de definir los métodos de explicación de los sistemas y asegurar un proceso específico para la gobernanza de la rendición de cuentas.

El Capítulo III proporciona una lista para la evaluación concreta pero no exhaustiva que debe adaptarse a los usos específicos.

Entre el 26 de junio y el 1 de diciembre de 2019, se desarrolló una fase piloto en la que las empresas podían testar la lista de evaluación y hacer comentarios y sugerencias además de compartir buenas prácticas para revisar las Directrices en 2020 si fuera necesario. Sin duda, se trata de un texto susceptible de mejora, que ha sido criticado por

\footnotetext{
Kaminski, M. E. (2019).

Von der Leyen, U. (2019): 14.

48 European Group on Ethics in Science and New Technologies (2018). En él, se establecían los principios éticos y requisitos democráticos que debían ser considerados para la creación de nuevos estándares sobre inteligencia artificial como: la dignidad humana; la autonomía; la responsabilidad; la justicia, equidad y solidaridad; la democracia; el estado de derecho; la sostenibilidad; la seguridad, integridad física y mental de las personas; y la protección de datos personales y la privacidad.

La Agencia de los Derechos Fundamentales de la UE también ha empezado a investigar el impacto de la inteligencia artificial en el disfrute de los derechos y ha identificado la lucha contra la discriminación, como un área particularmente afectada por los avances tecnológicos. En su opinión, cuando se utilizan algoritmos para la toma de decisiones, existe un potencial de discriminación contra los individuos, lo que sería contrario al artículo 21 de la Carta de los Derechos Fundamentales de la UE, Agencia de los Derechos Fundamentales de la Unión Europea (2018).

49 Su presidente (que proviene del mundo empresarial) se mostró reacio a regular la inteligencia artificial de un modo prematuro para no limitar la innovación y competitividad en la materia. En su opinión, Europa debería proponer principios horizontales amplios para el uso ético de la inteligencia artificial basados en los valores fundamentales europeos, pero no crearse una regulación genérica sino más bien ir adaptando la legislación a medida que surjan los problemas y se planteen casos ante los tribunales (Delcker, J., 2018), aunque podría argumentarse que esto puede chocar con un valor europeo fundamental como es la seguridad jurídica.

50 High-Level Expert Group on Artificial Intelligence (2019b).

51 Con la finalidad de definir qué es una IA robusta, las Directrices aclaran que se deberían prever medidas de protección para evitar cualquier efecto adverso imprevisto. Esto es necesario tanto desde un punto de vista técnico (garantizando una adecuada solidez técnica del sistema), como también social (teniendo debidamente en cuenta el contexto y el entorno en el que opera el sistema).
} 
múltiples actores ${ }^{52}$. El Grupo de Expertos de Alto Nivel también publicó el 26 de junio de 2019 una Recomendaciones de política e inversión para una IA fiable ${ }^{53}$.

\section{Posibilidades de cooperación entre Japón y la Unión Europea}

La UE y Japón comparten muchos de sus problemas dado que tienen una sociedad altamente envejecida ${ }^{54}$ y un nivel de vida elevado que no les permite competir con otras economías en el precio de la mano de obra, también dependen del exterior en las fuentes de energía y la obtención de muchas materias primas. Para poder seguir financiando el estado del bienestar deben especializarse en bienes y servicios con un alto valor añadido. Eso significa no perder la carrera en el desarrollo y control de la IA, pero ambos se han dado cuenta de que China y Estados Unidos les están dejando atrás ${ }^{55}$.

Margrethe Vestager, en su audiencia ante el Parlamento Europeo antes de convertirse en la Vicepresidenta de la Comisión Europea encargada de asegurar que Europa está preparada para la Era Digital, explicó que algunos dicen que los chinos tienen todos los datos y los estadounidenses tienen todo el dinero, pero Europa tiene un propósito, y que las Directrices para una IA fiable es lo que distinguía a Europa como un auténtico líder ${ }^{56}$. Japón ha decidido tomar un camino muy similar con sus Principios para una IA centrada en las personas.

Esto viene marcado porque la UE y Japón, en principio, comparten los mismos valores de respeto por la democracia, el estado de derecho y los derechos humanos ${ }^{57}$. Esto se ha plasmado en sus guías éticas que si bien no son idénticas son muy similares ${ }^{58}$, ya que ambas propugnan que el uso de la IA debe contribuir al bienestar social y centrarse en las personas, para lo que se plantean principios como la seguridad, la privacidad, la transparencia, la responsabilidad, la equidad y la rendición de $\operatorname{cuentas}^{59}$. Esta es una aproximación que diverge seriamente de la de países como China con su mecanismo de vigilancia masiva y sistema de crédito social.

En los últimos años han surgido cientos de guías éticas que describen los principios que deben regular el desarrollo de la $\mathrm{IA}^{60}$, una aproximación que tiene ciertos detractores ${ }^{61}$. Sin embargo, las de Japón y la UE pueden tener especial relevancia por varias razones. Por una parte, dada la importancia de ambas economías y porque ambos tienen como objetivo que sirvan como base a una discusión a nivel global sobre la materia. Japón ha sido muy persistente en las

52 Veale, M. (2020) critica que el documento es un maratón de recomendaciones, sin una priorización clara, muchas de ellas inútiles, confusas o no accionables, e ignoran en gran medida la infraestructura y el poder, que debería ser una de las principales preocupaciones, si no la más importante, en torno a la regulación y la gobernanza de los datos, la optimización y la IA en Europa en el futuro. Klöver, C./Fanta, A. (2019) critican que, desafortunadamente, no se pueden encontrar principios éticos inalterables en el texto final porque los representantes de la industria (Nokia, IBM, SAP) en el comité los han eliminado con éxito. Davola, A. et al. (2019)_critican que el documento pasa por alto la posible disyuntiva entre las pautas éticas idealistas y su implementación práctica: la ética en el terreno. Porque las listas de verificación como método de control no sirven para decidir si se debe construir algo o imaginar cómo se puede hacer éticamente, y tampoco sirven para lidiar con la ambigüedad. Por lo tanto, en su opinión, si bien las Directrices pueden agregar "valor ético", no imponen requisitos suficientemente específicos y consecuentes para crear confianza.

53 High-Level Expert Group on Artificial Intelligence (2019c).

54 Naciones Unidas (2019) aporta algunos datos muy reveladores. Japón registró 2,6 millones más de muertes que nacimientos entre 2010 y 2019 (p. 12). Los países de baja fertilidad ahora incluyen a toda Europa y Japón (p. 27). Con una expectativa de vida al nacer superior a 84 años en 2019 , Japón es el país más longevo del mundo (p. 30). En Europa existe una gran disparidad, con una esperanza de vida al nacer en 2019 que va desde 72 años en la República de Moldavia y Ucrania, hasta cerca de 84 años en Italia, España y Suiza (p. 31). Todo esto tiene como consecuencia que, con 1.8, Japón en 2019 tiene el índice de apoyo potencial más bajo de todos los países. El índice de apoyo potencial es el número de personas en edad de trabajar (25 a 64 años) por persona de 65 años o más. Otros 29 países, principalmente en Europa, tienen índices por debajo de tres. Para 2050, se espera que 48 países, principalmente en Europa, tengan índices de apoyo potenciales por debajo de dos (p. 20). Si se consultan los datos de la OECD (2019) de porcentaje de población mayor (65 años o más), Japón es el país con un porcentaje más elevado 24,15\%, pero los siguientes Estados pertenecen a la UE Alemania, Italia, Grecia, Suecia, Finlandia, Portugal, Letonia, Estonia, Austria y España.

55 Joint Research Centre (2018); Digital Transformation Monitor... (2018) y Allison, G. (2019).

56 www.europarl.europa.eu (2019).

57 Gascón Marcén, A. (2014).

58 Para una comparación más pormenorizada entre las dos Kozuka, S. (2019).

59 En el Berkman Klein Center for Internet \& Society de la Facultad de Derecho de Harvard, han desarrollado el Principled Artificial Intelligence Project que buscaba analizar las diferentes Guías Éticas que se están desarrollando no sólo por los Estados, sino también por la sociedad civil, las organizaciones internacionales, etc. y han concluido que hay ocho temas clave que se repiten normalmente en los mismos: responsabilidad; equidad y no discriminación; control humano de la tecnología; privacidad; responsabilidad profesional; promoción de valores humanos; seguridad y transparencia; y explicabilidad. Hilligoss, H. (2019). Floridi, L. et al. (2018) también intentaron buscar los elementos comunes de las listas de principios que concluyeron que se correspondían con los cuatro principios básicos de la bioética: autonomía, no maleficencia, beneficencia y justicia, a los que debía sumarse la explicabilidad.

60 En diciembre 2019, la Agencia de los Derechos Fundamentales de la UE hizo una recopilación de las iniciativas políticas relacionadas con la IA y consideró que sólo contando las que se han dado a nivel nacional en Estados europeos, u organizaciones de los que estos son miembros existían más de 260. Una "iniciativa política" se definió en términos generales para incluir una gama de iniciativas que podrían contribuir a la formulación de políticas y el establecimiento de normas en el área de la IA, desde legislación, directrices y recomendaciones sobre el uso de IA, o incluso informes que incluyen conclusiones con relevancia política. www.fra.europa.eu (2019).

${ }_{61}$ Las mayores críticas vienen de aquellos que consideran que en ocasiones la tendencia a elaborar marcos éticos para la IA viene promovida por la industria que los prefiere al desarrollo de legislación que es jurídicamente vinculante. Wagner, B. (2018) y Ochigame, R. (2019).

Mittelstadt, B. (2019) critica que la ética de la IA aparentemente ha convergido en un conjunto de principios que se parecen mucho a los cuatro principios clásicos de la ética médica, pero argumenta que, en comparación con la medicina, el desarrollo de IA carece de objetivos comunes y deberes fiduciarios, historia y normas profesionales, métodos probados para traducir principios en práctica y mecanismos sólidos de responsabilidad legal y profesional. En su opinión, estas diferencias sugieren que aún no deberíamos celebrar el consenso en torno a principios de alto nivel que ocultan un profundo desacuerdo político y normativo. 
discusiones en el G7 y en el G20, y la UE tiene un gran poder normativo capaz de crear estándares en materia de tecnologías de la información y la comunicación con impacto mundial dado el interés de las empresas de acceder al mercado único digital como lo ha demostrado con el Reglamento General de Protección de Datos ${ }^{62}$.

La colaboración en esta materia sería una buena manera de dar contenido al Acuerdo de Asociación Estratégica entre la Unión Europea y sus Estados miembros, por una parte, y Japón, por otra ${ }^{63}$, hecho en Tokio el 17 de julio de $2018^{64}$. En su artículo 21, las Partes se comprometieron a cooperar en políticas y normas en materia de tecnologías de la información y la comunicación, en especial en cuestiones esenciales, tales como las comunicaciones electrónicas, incluidas la gobernanza de internet y la seguridad en línea; la interconexión de las redes de investigación, sobre todo en un contexto regional; la promoción de las actividades de investigación e innovación; y la normalización y difusión de nuevas tecnologías. A esto se suma que la Comunidad Europea y el Gobierno de Japón celebraron un Acuerdo de Cooperación Científica y Tecnológica que entró en vigor en marzo de 2011.

En la Declaración conjunta de la vigésimo sexta Cumbre UE-Japón celebrada en Bruselas, el 25 de abril de $2019^{65}$, ambos se comprometieron a trabajar juntos, incluido en el marco del G7 y el G20, para promover un enfoque centrado en el ser humano para la inteligencia artificial. Con esa voluntad, se celebró una reunión el 3 de mayo de 2019 en Bruselas, entre Andrus Ansip, Vicepresidente de la Comisión Europea para el Mercado Único Digital, y Carlos Moedas, Comisario de Investigación, Ciencia e Innovación, y Hirai Takuya, Ministro de Estado de Política de Ciencia y Tecnología de Japón. En ella, se subrayó que éste es un tema clave en la cooperación entre la UE y Japón ${ }^{66}$. En concreto, se debatió sobre el potencial de la cooperación en materia de investigación. El programa Horizonte Europa de la UE y Moonshot de Japón buscan ambos responder a los grandes retos sociales fomentando la innovación, pero desde una perspectiva ética, por lo que sería interesante avanzar en la cofinanciación de proyectos conjuntos. Una posibilidad que se está barajando es que Japón se convierta en miembro asociado del programa Horizonte Europa al que contribuiría con 10 millones de euros al año para que sus investigadores pudieran participar más fácilmente en el mismo ${ }^{67}$. Una de las áreas prioritarias de cooperación sería la IA. Además, en 2018, se firmó un acuerdo para proporcionar oportunidades de colaboración entre investigadores del Consejo Europeo de Investigación y la Agencia de Ciencia y Tecnología de Japón ${ }^{68}$.

\section{Conclusiones}

Es necesario que se eleve la financiación de la investigación de la IA en la UE y Japón, puesto que China y Estados Unidos van muy por delante. Fomentar la investigación conjunta es una buena idea que no debería frenarse por el elevado nivel de burocratización de la UE. Hay que promover no sólo la inversión pública sino también la privada y el espíritu emprendedor, así como mejorar el marco para crear empresas de innovación y apoyar la formación en IA ${ }^{69}$.

La UE y Japón pueden aprender mutuamente de las medidas legislativas para fomentar la innovación, como la excepción para minería de textos y datos de Japón ${ }^{70}$, y a la par tomar medidas que protejan a las personas, como el principio de explicabilidad del Reglamento General de Protección de Datos de la UE¹.

\footnotetext{
Fefer, R. F. (2019).

3 www.eur-lex.europa.eu (2018); Gascón Marcén, A. (2019).
}

${ }_{64}$ Actualmente, el Acuerdo se aplica provisionalmente pendiente de su entrada en vigor para lo que se requiere la ratificación de todos los Estados miembros de la UE.

La UE y Japón también celebraron en paralelo un Acuerdo de Asociación Económica que está en vigor desde el 1 de febrero de 2019 y ha atraído la mayoría de la atención de la doctrina, Cisneros Cristóbal, P. (2019), Claudia Suciu, D. (2019) y Tirado Robles, C. (2020). Este Acuerdo, si bien no tiene un apartado dedicado a la inteligencia artificial, sí que contiene un artículo con un profundo impacto en la misma, el 8.73 que regula que una Parte no podrá exigir la transferencia del código fuente del software propiedad de una persona de la otra Parte ni el acceso a él, aunque esto no impedirá la inclusión o aplicación de términos y condiciones en relación con la transferencia o la concesión del acceso al código fuente en contratos negociados comercialmente, o la transferencia o concesión voluntaria del acceso al código fuente, por ejemplo, en el contexto de la contratación pública. Esta norma tampoco afectará a los requerimientos de un órgano jurisdiccional, un tribunal administrativo o una autoridad administrativa o de la competencia para subsanar una infracción del Derecho de la competencia o la protección y la aplicación de los derechos de propiedad intelectual, en la medida en que dichos códigos fuente estén protegidos por tales derechos.

Este artículo es una manifestación de la diferencia de las políticas de la UE y Japón frente a la mantenida, por ejemplo, por China, que en muchos casos para entrar en su mercado exige ese tipo de transferencias o que las empresas europeas tengan que buscar un socio local y así se les obliga a compartir su know-how. No obstante, el hecho de que sólo se creen excepciones para salvaguardar el Derecho de la competencia y la propiedad intelectual da la impresión de que se ha tenido una visión puramente economicista. Ese tipo de excepciones deberían existir también para proteger los derechos humanos, porque, por ejemplo, puede ser difícil demostrar que un algoritmo tiene un sesgo de discriminación si no existen mecanismos que permitan acceder a su código fuente (Irion, K. / Williams, J., 2019: 31 y 32).

65 www.consilium.europa.eu (2019a).

66 Hirai, Takuya (2019).

67 Hudson, R. L. (2019).

68 www.ec.europa.eu (2018).

${ }_{69}$ El programa de formación en IA japonés podría replicarse en la UE. Dentro de ésta también existen ya iniciativas interesantes como el programa finlandés de formación en IA que se creó como un curso en línea gratuito cuyo objetivo era que al menos un 1\% de la población del país conociera los principios básicos del funcionamiento de la IA y que se va a traducir a todas las lenguas oficiales de la UE, Delcker J. (2019).

70 La UE ha introducido recientemente en su legislación dos nuevas excepciones para la minería de textos y datos con la Directiva (UE) 2019/790 del Parlamento Europeo y del Consejo, de 17 de abril de 2019, sobre los derechos de autor y derechos afines en el mercado único digital y por la que se modifican las Directivas 96/9/CE y 2001/29/CE, DOUE L 130 de 17.5.2019, pp. 92-125. Sin embargo, las excepciones europeas son mucho más limitadas que la de la legislación japonesa que es más propicia a la innovación.

71 Gascón Marcén, A. (2020). 
Japón debería seguir de cerca la propuesta de legislación que publicará en 2020 la Comisión Europea con un enfoque europeo coordinado sobre las implicaciones éticas y humanas de la IA y, en el marco del Consejo de Europa, la negociación sobre un posible acuerdo internacional para la regulación de la IA desde una perspectiva de derechos humanos.

El hecho de que la UE y Japón hayan declarado que la cooperación en materia de IA es prioritaria es muy positivo y puede darle un contenido tangible al Acuerdo Estratégico UE-Japón. Sin embargo, como siempre en estas relaciones, hay que ver cómo pasar de las palabras y el potencial a los resultados ${ }^{72}$.

La UE y Japón son aliados naturales para defender a nivel internacional una IA centrada en las personas y que respete los derechos humanos ${ }^{73}$. Según las directrices desarrolladas por ambos, está claro que hay que seguir fomentando la transparencia, la rendición de cuentas, la seguridad y la privacidad.

La UE y Japón deben promocionar las ventajas de su aproximación a la IA desde la óptica de su aportación a mejorar la vida de las personas y reducir el impacto negativo que puedan tener en los derechos humanos. Para ello, deben conseguir nuevos aliados en los foros internacionales, ya que se trata de un tema con un profundo impacto geoestratégico ${ }^{74}$. No puede olvidarse que la aproximación china que obvia ese tipo de riesgos tiene una fuerza atractiva internacional ${ }^{75}$. También deberían plantearse fijar algunas líneas rojas sobre qué tipo de actividades no deben confiarse a la IA.

Para concluir, debe resaltarse que ha llegado el momento de ir más allá de los principios y ver cómo implementar éstos en la práctica a través de: reformas legislativas creando obligaciones jurídicamente vinculantes, cuando sea necesario, formación de los desarrolladores, y políticas de financiación de la investigación.

\section{Bibliografía}

Allison, Graham (2019): “Is China Beating America to AI Supremacy?”, The National Interest, 22/12/2019. https:// nationalinterest.org/feature/china-beating-america-ai-supremacy-106861 [Consulta: 29/12/2019].

Alston, Philip (2019): Informe del Relator Especial sobre la extrema pobreza y los derechos humanos a la Asamblea General de las Naciones Unidas, 11 de octubre de 2019, A/74/493. United Nations Documents. https://undocs.org/ pdf?symbol=es/A/74/493 [Consulta: 29/12/2019].

Berkofsky, Axel (2018): The EU-Japan Strategic Partnership Agreement (SPA): Good but Good Enough? Italian Institute for International Political Studies. https://www.ispionline.it/en/pubblicazione/eu-japan-strategic-partnershipagreement-spa-good-good-enough-21392 [Consulta: 29/12/2019].

Borgesius, Frederik Zuiderveen (2018): Discrimination, artificial intelligence, and algorithmic decision-making. Council of Europe. https://rm.coe.int/discrimination-artificial-intelligence-and-algorithmic-decision-making/1680925d73 [Consulta: 29/12/2019].

Cisneros Cristóbal, Paula (2019): "El acuerdo de asociación económica entre la Unión Europea y Japón: negociación, contenido y análisis de los elementos clave”. En: Derecho y relaciones internacionales en Japón: Desde el Tratado de Amistad, Comercio y Navegación de 1868. Zaragoza: Prensas de la Universidad de Zaragoza, pp. 173-210.

\footnotetext{
Berkofsky, A. (2018).
}

73 Pueden apoyarse en el trabajo del Relator Especial de Naciones Unidas (Kaye, D., 2018), que propone medidas concretas como son: las evaluaciones de impacto de la inteligencia artificial sobre los derechos humanos; las auditorías externas que presentan problemas al enfrentarse a algoritmos protegidos pero que podrían elaborarse incluso sin acceso directo al código (por ejemplo, a través de zero-knowledge proofs) o permitiéndoselo sólo a algunos terceros certificados; y los mecanismos de refuerzo de la autonomía individual. Además, el Relator considera esencial asegurar medidas correctivas cuando se comprueben violaciones de los derechos humanos.

74 Ortega Klein, A. (2020).

75 China es el mayor impulsor de la vigilancia basada en IA en todo el mundo. Según Feldstein (2019: 1 y 2), la tecnología vinculada a las empresas chinas, en particular Huawei, Hikvision, Dahua y ZTE, suministra tecnología de vigilancia de IA en sesenta y tres países, treinta y seis de los cuales han firmado la Belt and Road Initiative de China. Solo Huawei es responsable de proporcionar tecnología de vigilancia de IA al menos a cincuenta países de todo el mundo. Ninguna otra compañía se acerca. Sin embargo, cabe destacar que el siguiente mayor proveedor no chino de este tipo de tecnología es la Corporación NEC de Japón (catorce países). Otro elemento preocupante, según este autor, es que los lanzamientos de productos chinos a menudo van acompañados de préstamos blandos para alentar a los gobiernos a comprar sus equipos. Estas tácticas son particularmente relevantes en países como Kenia, Laos, Mongolia, Uganda y Uzbekistán, que de otro modo no podrían acceder a esta tecnología, esto supone que el gobierno chino está subsidiando la compra de tecnología represiva avanzada. Sin embargo, esta cuestión es más compleja de lo que puede parecer puesto que no se puede demonizar simplemente a China, porque no es el único país que suministra tecnología de vigilancia avanzada en todo el mundo. Otras compañías basadas en democracias liberales como Estados Unidos, pero también Francia, Alemania o Japón, están desempeñando papeles importantes en la proliferación de esta tecnología. El autor considera, por tanto, que democracias (como las de Japón o la UE) no están tomando los pasos adecuados para vigilar y controlar la difusión de tecnologías sofisticadas vinculadas a una variedad de violaciones de derechos humanos. Véase también Polyakova y Meserole, 2019

El propio Relator Especial de Naciones Unidas sobre la promoción y protección del derecho a la libertad de opinión y de expresión hizo en 2019 un llamamiento a la imposición de una regulación más rigurosa de las exportaciones de equipos de vigilancia y unas restricciones más estrictas de su utilización, así como de una moratoria inmediata sobre la venta y la transferencia a nivel mundial de los instrumentos que utiliza el sector de la vigilancia privada hasta que se establezcan estrictas salvaguardias de los derechos humanos en la regulación de esas prácticas y se pueda garantizar que los gobiernos y los agentes no estatales van a utilizar esos instrumentos de un modo legítimo (Kaye, D. 2019: 1). En ese sentido, la Comisión Europea adoptó en 2016 una Propuesta de Reglamento del Parlamento Europeo y del Consejo por el que se establece un régimen de la Unión de control de las exportaciones, la transferencia, el corretaje, la asistencia técnica y el tránsito de productos de doble uso. E1 Parlamento Europeo se mostró partidario de controlar las exportaciones de programas de vigilancia cibernética por motivos de derechos humanos, pero varios Estados en el seno del Consejo no estaban de acuerdo (Kanetake, M. 2019). 
Clarke, Roger (2019): Evaluation of the OECD's AI Guidelines. Roger Clarke's Web-Site. http://www.rogerclarke.com/ EC/AI-OECDEval.html [Consulta: 29/12/2019].

Davola, Antonio / Black, Emily / Gulson, Kalervo / Rockwell, Geoffrey / Selinger, Evan / Zeide, Elana (2019): Shortcut or Sleight of Hand? Why The Checklist Approach in The EU Guidelines Does Not Work. AIPulse https://aipulse. org/from-shortcut-to-sleight-of-hand-why-the-checklist-approach-in-the-eu-guidelines-does-not-work/ [Consulta: 29/12/2019].

Delcker, Janosch (2018): Europe's AI ethics chief: No rules yet, please. Politico.eu, 30/09/2018. https://www.politico. eu/article/pekka-ala-pietila-artificial-intelligence-europe-shouldnt-rush-to-regulate-ai-says-top-ethics-adviser/ [Consulta: 29/12/2019].

Delcker, Janosch (2019): Finland's grand AI experiment. Politico.eu, 01/02/19 https:/www.politico.eu/article/finlandone-percent-ai-artificial-intelligence-courses-learning-training/ [Consulta: 29/12/2019].

Digital Transformation Monitor. USA-China-EU plans for AI: where do we stand? (2018). European Commission. https:// ec.europa.eu/growth/tools-databases/dem/monitor/content/usa-china-eu-plans-ai-where-do-we-stand [Consulta: 29/12/2019].

Dilsizian, Steven E. / Siegel, Eliot L. (2014): Artificial intelligence in medicine and cardiac imaging: harnessing big data and advanced computing to provide personalized medical diagnosis and treatment. En: Current cardiology reports, Vol. 16, No 1, artículo 441. < doi:10.1007/s11886-013-0441-8>.

European Group on Ethics in Science and New Technologies (2018): "Artificial Intelligence, Robotics and 'Autonomous' Systems”. Luxemburgo: Oficina de Publicaciones de la Unión Europea, <doi:10.2777/531856>.

Fefer, Rachel F. (26 de marzo 2020): Data Flows, Online Privacy, and Trade Policy. Congressional Research Service https://crsreports.congress.gov/product/pdf/R/R45584 [Consulta: 29/12/2019].

Feldstein, Steven (2019): The Global Expansion of AI Surveillance. Carnegie Endowment for International Peace. https:// carnegieendowment.org/files/WP-Feldstein-AISurveillance_final1.pdf [Consulta: 29/12/2019].

Floridi, Luciano / Cowls, Josh / Beltrametti, Monica / Chatila, Raja / Chazerand, Patrice / Dignum, Virginia / Luetge, Christoph / Madelin, Robert / Pagallo, Ugo / Rossi, Francesca / Schafer, Burkhard / Valcke Peggy / Vayena, Effy (2018): "AI4People-An Ethical Framework for a Good AI Society: Opportunities, Risks, Principles, and Recommendations." En: Minds \& Machines vol. 28, pp. 689-707 < doi:10.1007/s11023-018-9482-5>.

Gascón Marcén, Ana (2014): “Japón y el Consejo de Europa. Algunas reflexiones sobre los valores compartidos y la pena de muerte.” En: Tirado Robles, María Carmen (ed.). Japón y Occidente: estudios comparados. Zaragoza: Prensas Universitarias de Zaragoza, pp. 113-132.

Gascón Marcén, Ana (2016): "La nueva protección de datos personales: una mirada a Japón desde Europa". En: Tirado Robles, María Carmen / Barberán Pelegrín, Francisco (eds.). Los derechos individuales en el ordenamiento japonés. Madrid: Thomson Reuters Aranzadi, pp. 129-148.

Gascón Marcén, Ana (2019): "Las relaciones entre Japón y la Unión Europea: pasos hacia una auténtica relación estratégica”. En: Tirado Robles, María Carmen / Barberán Pelegrín, Francisco (eds.). Derecho y relaciones internacionales en Japón: Desde el Tratado de Amistad, Comercio y Navegación de 1868. Zaragoza: Prensas Universitarias de Zaragoza, pp. 211-244.

Gascón Marcén, Ana (2020): Society 5.0: EU-Japanese cooperation and the opportunities and challenges posed by the data economy. ARI 11/2020. Real Instituto Elcano, Strategic and International Studies. http://www.realinstitutoelcano. org/wps/portal/rielcano_en/contenido?WCM_GLOBAL_CONTEXT=/elcano/elcano_in/zonas_in/ari11-2020gascon-society-5-0-eu-japanese-cooperation-and-opportunities-and-challenges-posed-by-data-economy [Consulta: 4/2/2020].

Greenleaf, Graham (2019): "G20 makes declaration of 'Data Free Flow with Trust': Support and Dissent”. En: 160 Privacy Laws \& Business International Report, pp. 18-19. https://papers.ssrn.com/sol3/papers.cfm?abstract $\mathrm{id}=3514407 \&$ download=yes [Consulta: 29/12/2019].

High-Level Expert Group on Artificial Intelligence (2019a): A definition of AI: main capabilities and disciplines. European Council. https://ec.europa.eu/digital-single-market/en/news/definition-artificial-intelligence-main-capabilities-andscientific-disciplines [Consulta: 29/12/2019].

High-Level Expert Group on Artificial Intelligence (2019b): Ethics guidelines for trustworthy AI. European Council. https://ec.europa.eu/digital-single-market/en/news/ethics-guidelines-trustworthy-ai [Consulta: 29/12/2019].

High-Level Expert Group on Artificial Intelligence (2019c): Policy and investment recommendations for trustworthy AI. European Council. https://ec.europa.eu/digital-single-market/en/news/policy-and-investment-recommendationstrustworthy-artificial-intelligence [Consulta: 29/12/2019].

Hilligoss, Hannah (7 de junio 2019): Introducing the Principled Artificial Intelligence Project. Cyberlaw Clinic. Harvard Law School/ Berkman Klein Center for Internet \& Society. https:/clinic.cyber.harvard.edu/2019/06/07/introducingthe-principled-artificial-intelligence-project/ [Consulta: 29/12/2019].

Hirai, Takuya (2019): Cooperation with America and Europe regarding "the Moonshot Research and Development Program" (Report of business trip to America and Europe, made by Mr. Takuya Hirai, Minister of State for Science and Technology Policy). Cabinet Office Government of Japan. https://www8.cao.go.jp/cstp/english/moonshot/3rd/ doc1-1.pdf [Consulta: 29/12/2019].

Hornyak, Tim (1 de agosto 2017): Japan shakes up research funding system. Nature Index. https://www.natureindex.com/ news-blog/japan-shakes-up-research-funding-system [Consulta: 29/12/2019]. 
Hudson, Richard L. (6 de noviembre 2019): Japan eyes possible $€ 10 \mathrm{M}$ a year for Horizon Europe partnerships. Science Business. https://sciencebusiness.net/framework-programmes/news/japan-eyes-possible-eu10m-year-horizoneurope-partnerships [Consulta: 29/12/2019].

Inagaki, Kana (18 de Julio 2019): Japan falling behind in artificial intelligence, warns SoftBank founder. Financial Times. https://www.ft.com/content/cab0936c-a940-11e9-984c-fac8325aaa04 [Consulta: 29/12/2019].

Irion, Kristina / Williams, Josephine (2019): Prospective Policy Study on Artificial Intelligence and EU Trade Policy. Amsterdam: The Institute for information Law.

Jacob, Michael (15 de abril 2019): Japan increasing R\&I budget more than 10 percent, and shooting at the moon. Offices of Science and Innovation. https://sweden-science-innovation.blog/japan/japan-increasing-ri-budget-more-than-10percent-and-shooting-at-the-moon/ [Consulta: 29/12/2019].

Jin, Xu / Wang, Dianhong. (2008): “An intelligent model for urban demand-responsive transport system control”. En: 2008 International Symposium on Intelligent Information Technology Application Workshops. New York: Institute of Electrical and Electronic Engineers, pp. 151-154. <doi:10.1109/IITA.Workshops.2008.62>.

Joint Research Centre (2018): Artificial Intelligence. A European perspective. Luxemburgo: Oficina de Publicaciones de la Unión Europea. < doi:10.2760/936974>

Kaminski, Margot E. (2019): “The Right to Explanation, Explained”. En: Berkeley Technology Law Journal, Vol. 34 , No. 1, pp. 189-218< doi:10.2139/ssrn.3196985>.

Kanetake, Machiko (2019): 'The EU's dual-use export control and human rights risks: the case of cyber surveillance technology”. En: Europe and the World: A law review, Vol. 3, No. $1<$ https://doi.org/10.14324/111.444.ewlj.2019.14>.

Kaye, David (2018): Informe del Relator Especial sobre la promoción y protección del derecho a la libertad de opinión y de expresión, Asamblea General de Naciones Unidas, A/73/348. United Nations Documents. https://undocs.org/ pdf?symbol=es/A/73/348 [Consulta: 29/12/2019].

Kaye, David (2019): Informe del Relator Especial sobre la promoción y protección del derecho a la libertad de opinión y de expresión. La vigilancia y los derechos humanos, A/HRC/41/35. United Nations Documents https://documentsdds-ny.un.org/doc/UNDOC/GEN/G19/148/79/PDF/G1914879.pdf?OpenElement [Consulta: 29/12/2019].

Klöver, Chris / Fanta, Alexander (9 de abril 2019): No red lines: Industry defuses ethics guidelines for artificial intelligence. AlgorithmWatch https://algorithmwatch.org/en/industry-defuses-ethics-guidelines-for-artificial-intelligence/ [Consulta: 29/12/2019].

Kozuka, Souichirou (2018): “Japan's Response to New Technologies: Draft Artificial Intelligence Research and Development Guidelines for International Discussions”. En: Zeitschrift für Japanisches Recht (Journal of Japanese Law), Vol. 23, No. 46, pp. 3-18.

Kozuka, Souichirou (2019): “A governance framework for the development and use of artificial intelligence: lessons from the comparison of Japanese and European initiatives". En: Uniform Law Review, Vol. 24, No. 2, pp. 315-329, $<$ doi:10.1093/ulr/unz014>.

McCarthy, John / Minsky, Marvin L. / Rochester, Nathaniel / Shannon, Claude E. (2006): “A Proposal for the Dartmouth Summer Research Project on Artificial Intelligence, August 31, 1955”. AI Magazine, Vol. 27, No. 4, 12-14, <doi: 10.1609/aimag.v27i4.1904>.

Millar, Jason / Barron, Brent / Hori, Koichi / Finlay, Rebecca / Kotsuki, Kentaro / Kerr, Ian (2018): Accountability in AI. Promoting Greater Social Trust. Theme Paper for the G7 Multi-stakeholder Conference on Artificial Intelligence: Enabling the Responsible Adoption of AI, December 6, 2018. Canadian Institute for Advanced Research https://www. cifar.ca/docs/default-source/ai-society/g7-accountabilityinai.pdf?sfvrsn=66bdfdc2_6 [Consulta: 29/12/2019].

Miller, Hannah / Stirling, Richard (2019): Government Artificial Intelligence Readiness Index 2019. Oxford Insights. https://www.oxfordinsights.com/ai-readiness2019 [Consulta: 29/12/2019].

Mittelstadt, Brent (2019): "Principles alone cannot guarantee ethical AI". En: Nature Machine Intelligence, Vol. 1, pp. 501-507. https://www.nature.com/articles/s42256-019-0114-4 [Consulta: 29/12/2019].

Ochigame, Rodrigo (2019): “The invention of "ethical AI" How Big Tech Manipulates Academia to Avoid Regulation”, The Intercept, 20/12/2019, https://theintercept.com/2019/12/20/mit-ethical-ai-artificial-intelligence/ [Consulta: 29/12/2019].

Oikawa, Akira (2019): “Japan's R\&D spending hits record for nearly half of companies”, Nikkei Asian Review, 14/09/2019 https://asia.nikkei.com/Business/Business-trends/Japan-s-R-D-spending-hits-record-for-nearly-half-of-companies [Consulta: 29/12/2019].

Ortega Klein, Andrés (2019): Sociedad 5.0: el concepto japonés para una sociedad superinteligente, Real Instituto Elcano, ARI 10/2019. Real Instituto Elcano, Strategic and International Studies. http://www.realinstitutoelcano.org/wps/ portal/rielcano_es/contenido?WCM_GLOBAL_CONTEXT=/elcano/elcano_es/zonas_es/economia+internacional/ ari10-2019-ortega-sociedad-5-0-concepto-japones-sociedad-superinteligente [Consulta: 29/12/2019].

Ortega Klein, Andrés (2020): Geopolítica de la ética en Inteligencia Artificial, Real Instituto Elcano, Documento de Trabajo 1/2020. Real Instituto Elcano, Strategic and International Studies. http://www.realinstitutoelcano.org/wps/portal/ rielcano_es/contenido?WCM_GLOBAL_CONTEXT=/elcano/elcano_es/zonas_es/dt1-2020-ortega-geopolitica-dela-etica-en-inteligencia-artificial [Consulta: 29/12/2019].

Park, Seong Ho / Han, Kyunghwa (2018): "Methodologic guide for evaluating clinical performance and effect of artificial intelligence technology for medical diagnosis and prediction”. En: Radiology, Vol. 286, No. 3, pp. 800-809. $<$ doi:10.1148/radiol.2017171920>. 
Patrício, Diego Inácio / Rieder, Rafael (2018): “Computer vision and artificial intelligence in precision agriculture for grain crops: A systematic review”. En: Computers and Electronics in Agriculture, Vol. 153, pp. 69-81. < doi:10.1016/j. compag.2018.08.001>.

Perrault, Raymond / Shoham, Yoav / Brynjolfsson, Erik / Clark, Jack / Etchemendy, John / Grosz, Barbara / Lyons, Terah / Manyika, James / Mishra, Saurabh / Niebles, Juan Carlos (2019): The AI Index 2019 Annual Report. Palo Alto: HumanCentered AI Institute, Stanford University. https://hai.stanford.edu/sites/g/files/sbiybj10986/f/ai_index_2019_report. pdf [Consulta: 29/12/2019].

Polyakova, Alina / Meserole, Chris (agosto 2019): Exporting digital authoritarianism. The Russian and Chinese models. Brookings. https://www.brookings.edu/research/exporting-digital-authoritarianism/ [Consulta: 29/12/2019].

Raso, Filippo / Hilligoss, Hannah / Krishnamurthy, Vivek / Bavitz, Christopher / Kim, Levin Yerin (2018): Artificial Intelligence \& Human Rights: Opportunities \& Risks, Berkman Klein Center Research Publication No. 2018-6. $<$ doi:10.2139/ssrn.3259344>.

Suciu, Dorina Claudia (2019): “El Acuerdo entre la Unión Europea y Japón relativo a una asociación económica: una gran apuesta por el libre comercio". En: Revista General de Derecho Europeo, No. 48, s/p. https://www.iustel.com/ v2/revistas/detalle_revista.asp?numero $=48 \& \mathrm{id}=13$

Tirado Robles, Carmen (2020): “El Acuerdo Unión Europea-Japón”. En: Blanc Altemir, Antonio (ed.). La Unión Europea, promotora del libre comercio. Análisis e impacto de los principales acuerdos comerciales. Madrid: Aranzadi, pp. 51-73.

Veale, Michael (24 de enero 2020): “A Critical Take on the Policy Recommendations of the EU High-Level Expert Group on Artificial Intelligence”. En: European Journal of Risk Regulation. < doi:10.31228/osf.io/dvx4f>.

Von der Leyen, Ursula (16 de julio 2019): Orientaciones políticas para la próxima Comisión Europea (2019-2024). Una Unión que se esfuerza por lograr más resultados: Mi agenda para Europa . European Council. https://ec.europa.eu/ commission/sites/beta-political/files/political-guidelines-next-commission_es.pdf [Consulta: 29/12/2019].

Wagner, Ben (2018): "Ethics as an escape from regulation: From ethics-washing to ethics-shopping.". En: Bayamlioglu, Emre / Baraliuc, Irina / Janssens, Liisa A. W. / Hildebrandt, Mireille (eds.). Being Profiled: Cogitas Ergo Sum. Amsterdam: University Press, Amsterdam, pp. 84-90<doi:10.2307/j.ctvhrd092.18>.

www.consilium.europa.eu (2019a): EU-Japan Summit Joint Statement (25 de abril 2019): European Council. https:// www.consilium.europa.eu/media/39222/190425-eu-japan_summit-statement-final.pdf [Consulta: 29/12/2019].

www.consilium.europa.eu (2019b): G20 Osaka Leaders' Declaration. European Council. https://www.consilium.europa. eu/media/40124/final_g20_osaka_leaders_declaration.pdf [Consulta: 29/12/2019].

www.data.oecd.org (2019): Elderly population. OECD Data. https://data.oecd.org/pop/elderly-population.htm [Consulta: 29/12/2019].

www.ec.europa.eu (2018): New cooperation arrangement signed between the European Commission and the Japan Science and Technology Agency to strengthen researcher's cooperation. European Commission. https://ec.europa.eu/ research/iscp/index.cfm?pg=news_by_country\&countryname=japan [Consulta: 29/12/2019].

www.eur-lex.europa.eu (2018): Acuerdo de Asociación Estratégica entre la Unión Europea y sus Estados miembros, por una parte, y Japón, por otra. ST/8463/2018/INIT. EUR-Lex. Access to European Union law. https://eur-lex.europa.eu/ legal-content/ES/TXT/?qid=1577560194271\&uri=CELEX:22018A0824(01) [Consulta: 29/12/2019].

www.europarl.europa.eu (2019): Hearing of Margrethe Vestager, at the Committee on Industry, Research and Energy, Committee on the Internal Market and Consumer Protection, Committee on Economic and Monetary Affairs (8 de octubre 2019). European Parliament. https://www.europarl.europa.eu/resources/library/media/20191009RES63801/20191009RES63801. pdf [Consulta: 29/12/2019].

www.fra.europa.eu (2019): AI policy initiatives (2016-2019). Artificial Intelligence and Big Data; and Fundamental Rights. FRA. European Union Agency for Fundamental Rights https://fra.europa.eu/en/project/2018/artificialintelligence-big-data-and-fundamental-rights/ai-policy-initiatives [Consulta: 29/12/2019].

www.fra.europa.eu (30 de mayo 2018): \#BigData: Discrimination in data-supported decision making: FRA. European Union Agency for Fundamental Rights. https://fra.europa.eu/en/publication/2018/big-data-discrimination [Consulta: 29/12/2019].

www.gsmaintelligence.com (2019): The Mobile Economy 2019. GSMA Intelligence https:/www.gsmaintelligence.com/ research/?file=b9a6e6202ee1d5f787cfebb95d3639c5\&download [Consulta: 29/12/2019].

www.legalinstruments.oecd.org (2019): OECD/LEGAL/0449. Recommendation of the Council on Artificial Intelligence. OECD Legal Instruments. https://legalinstruments.oecd.org/en/instruments/OECD-LEGAL-0449\#_ ga=2.86793752.189492959.1560336874-1504412241.1560336874 [Consulta: 29/12/2019].

www.mofa.go.jp (2019): G20 Ministerial Statement on Trade and Digital Economy. Ministry of Foreign Affairs of Japan. https://www.mofa.go.jp/files/000486596.pdf [Consulta: 29/12/2019].

www.nedo.go.jp (31 de marzo 2017): Artificial Intelligence Technology Strategy (Report of Strategic council for AI Technology). NEDO. https://www.nedo.go.jp/content/100865202.pdf [Consulta: 29/12/2019].

www.oecd.org (2018): OECD Science, Technology and Innovation Outlook 2018. Adapting to Technological and Societal Disruption. OECD. https://www.oecd.org/sti/oecd-science-technology-and-innovation-outlook-25186167. htm [Consulta: 29/12/2019].

www.population.un.org (2019): World Population Prospects 2019. Highlights. United Nations. Department of Economic and Social Affairs, Population Division. https://population.un.org/wpp/Publications/Files/WPP2019_Highlights.pdf [Consulta: 29/12/2019]. 
www.rm.coe.int (2017): Algorithms and Human Rights. Study on the human rights dimensions of automated data processing techniques and possible regulatory implications. Council of Europe. Committee of Experts on Internet Intermediaries. https://rm.coe.int/study-hr-dimension-of-automated-data-processing-incl-algorithms/168075b94a [Consulta: 29/12/2019].

www.soumu.go.jp (2017): Draft AI R\&D Guidelines for International Discussions (28 July 2017). The Conference toward AI Network Society. Ministry of Internal Affairs and Communications. https://www.soumu.go.jp/main content/000507517.pdf [Consulta: 29/12/2019].

www.soumu.go.jp (2018): Draft AI Utilization Principles (17 July 2018). The Conference toward AI Network Society. Ministry of Internal Affairs and Communications. https://www.soumu.go.jp/main_content/000581310.pdf [Consulta: 29/12/2019].

www8.cao.go.jp (2015): Report on The 5th Science and Technology Basic Plan (18 de diciembre 2015). Cabinet Office Government of Japan. https://www8.cao.go.jp/cstp/kihonkeikaku/5basicplan_en.pdf [Consulta: 29/12/2019].

www8.cao.go.jp (2017): Integrated Innovation Strategy. Cabinet Office Government of Japan. https://www8.cao.go.jp/ cstp/english/doc/integrated_main.pdf [Consulta: 29/12/2019].

www8.cao.go.jp (2018): Moonshot Research and Development Program. Cabinet Office Government of Japan. https:// www8.cao.go.jp/cstp/english/moonshot1.pdf [Consulta: 29/12/2019].

www8.cao.go.jp (2019): Social Principles of Human-centric AI. Cabinet Office Government of Japan https://www8.cao. go.jp/cstp/stmain/aisocialprinciples.pdf [Consulta: 29/12/2019].

Yamashita, Minako (30 de marzo 2019): Japan aims to produce 250,000 AI experts a year. Nikkei Asian Review. https:// asia.nikkei.com/Economy/Japan-aims-to-produce-250-000-AI-experts-a-year [Consulta: 29/12/2019]. 